\title{
A SIMPLE OPTICAL APPARATUS FOR MEASURING THE CORNEAL THICKNESS, AND THE AVERAGE THICKNESS OF THE HUMAN CORNEA*
}

\author{
BY \\ D. M. MAURICE AND A. A. GIARDINI $\dagger$ \\ Ophthalmological Research Unit, Medical Research Council, \\ Institute of Ophthalmology, London
}

Previous methods of measuring the corneal thickness in the living eye have recently been extensively reviewed and criticized by von Bahr (1948a), who devised an ingenious method (1948a, b) of overcoming the practical difficulties and theoretical objections of its forerunners. He employed an apparatus consisting of two rotating glass plates, coupled by gearing, which were mounted in front of the condensing lens and microscope of a slit lamp, so that the aperture of each was half covered by one of these plates. On rotation, the reflection of the slit in the corneal endothelium may be displaced in the field of view of the microscope and brought into coincidence with that in the epithelium. By noting the angle of rotation, the thickness of the cornea may be obtained from a curve derived by calculation. Von Bahr claimed for this instrument that it was simple and easy to handle and gave a satisfactory degree of accuracy and consistency.

We were projecting a series of experiments in which changes in the thickness of the cornea in rabbits were to be followed after its metabolism was interfered with in various ways. Accordingly, a similar apparatus was constructed and attached to a Haag-Streit slit lamp. Experience showed, however, that there were disadvantages in this arrangement which made it difficult to use with a normal eye, and quite impossible with disturbed corneae for which it had been intended.

There are eight images of the slit formed by this optical system when operating correctly, and in our model an extra "ghost" image due to a double reflection within the glass plates. Two of these images, one an epithelial, and one an endothelial reflex, have to be aligned, but this is not easy as the former is some thirty times the brighter, and its dazzle conceals the other when they are adjoining. Again, the closeness of the glass plates to the slit-lamp and microscope lenses resulted in the light which traversed the first

* Received for publication November 13, 1950.

+ Now at the Ophthalmic Clinic, University of Parma, Italy. 
plate, passing almost entirely over the second and vice versa, so that the reflections which have to be aligned are considerably less intense than the subsidiary ones; in fairness it must be admitted that this defect would be of minor importance in von Bahr's own apparatus, as there was greater space between the plate and the slit-lamp lens. Finally, it is inconvenient to have a reduction in the free space in front of the slit lamp and microscope.

We decided, therefore, to give up the model of the von Bahr apparatus and to design one which would be free from these objections. A simple and satisfactory device was finally constructed which could instantly be attached to the Haag-Streit slit lamp, or detached from it, leaving it free for normal use. This also appeared superior in performance to von Bahr's own instrument.

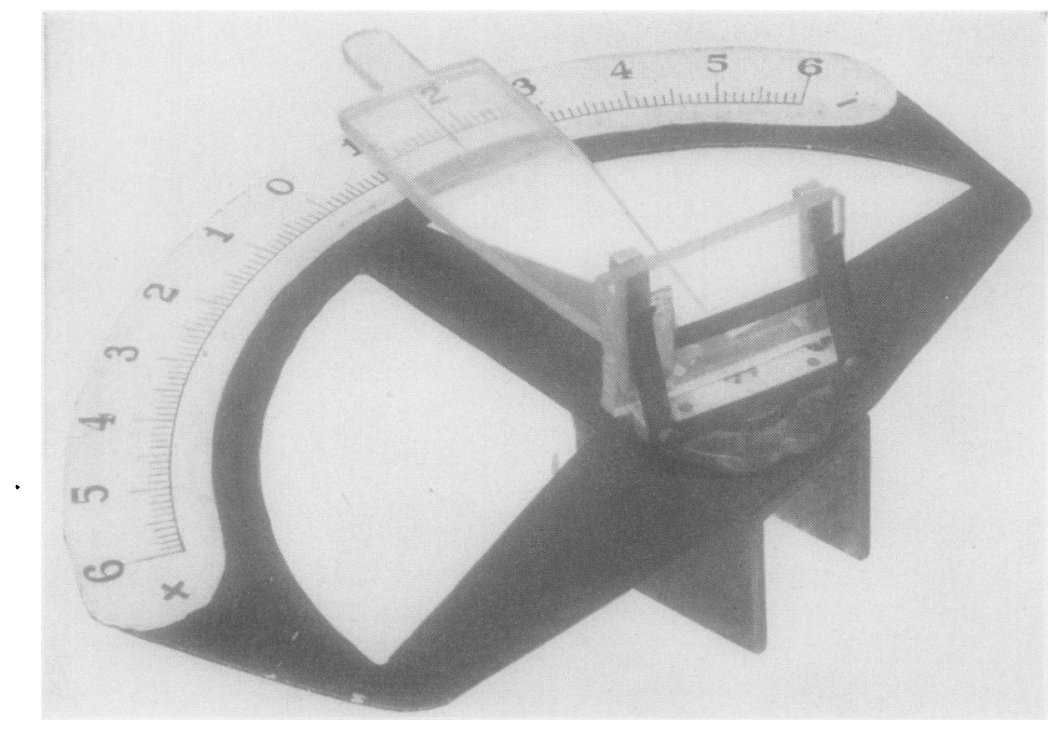

FIc;. 1.-General view of attachment.

Desicin

The principle of the apparatus is derived from that of von Bahr, but instead of two thin glass plates in front of the slit lamp, there is only one thick (t) in.) perspex plate mounted behind the slit-lamp lens, that is, between the lens and the slit. A cut about $1 \mathrm{~mm}$. wide is made centrally across this plate with a saw, and this is covered with a thin strip of coloured celluloid. The plate may be rotated by an arm which moves over a scale fixed to a mount fitting snugly over the slit-lamp arm, on which it is located by the two screws normally present (Fig. 1).

The optical principle is illustrated in Fig. 2. The slit lamp is adjusted so that a narrow slit is focused on the cornea, and the light, on reflection at the corneal surfaces, passes up the axis of the microscope. In this way two optical sections of the cornea will be seen on looking into the microscope, a normal " white " one which is displaced 
in the field of view as the perspex plate is rotated, and a less intense coloured one, virtually restricted to the epithelial reflex, which remains stationary. To carry out a determination, the plate is rotated until the white endothelial reflex is brought into alignment with the coloured epithelial reflex with which it is comparable in brightness, the angle of rotation being a measure of the corneal thickness.

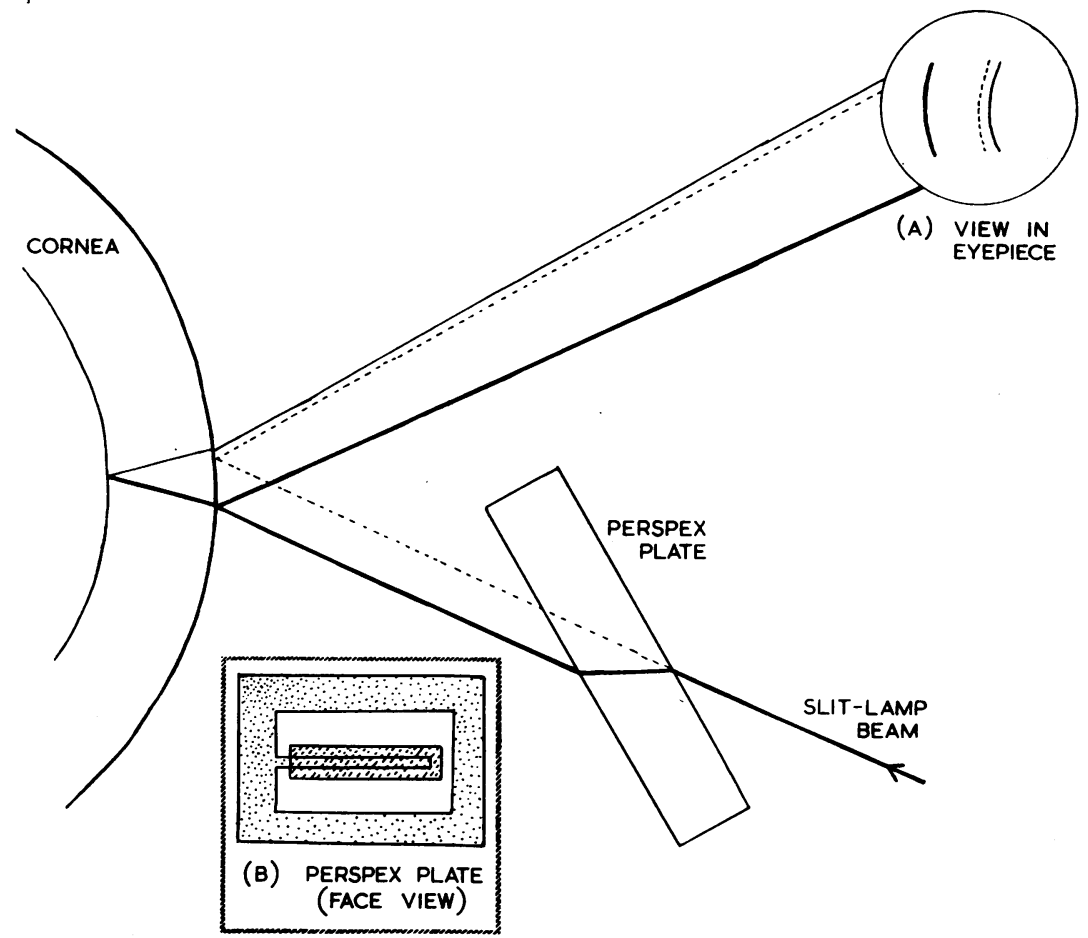

FIG. 2.-Diagram of path of rays, slit-lamp lens omitted for simplicity. Coloured light indicated by broken lines. (A) view in eyepiece with epithelial and endothelial reflections nearly in alignment. (B) face-on appearance of perspex plate with celluloid covering saw-cut.

\section{USE}

Only one eyepiece is required and full magnification ( $\mathrm{x} 40$ ) is convenient. The focusing controls on the slit-lamp lens and microscope are allowed to lock into the standard positions predetermined by the manufacturers. The arms of the slit lamp and microscope are fixed together with a definite angle, in our case $50^{\circ}$, between the beam and chosen objective. The slit is focused on the chosen area of the cornea, and the arms are rotated until the coloured epithelial reflection takes up a position in the centre of the diffuse patch of light that is the reflection of the slit-lamp lens in the cornea. In this position, which is reproducible with sufficient accuracy, the reflections in the corneal interfaces are near maximum brightness. 
The slit is then narrowed until the sharpness and brightness are optimum, and the appropriate reflections are aligned by adjusting the rotating arm on the attachment. With the other hand the focus and position of the images of the slit are maintained. The initial setting is achieved in about 30 seconds and repeat settings in 15 seconds.

For human subjects, the eye not being measured is made to fix an object seen in a mirror attached to the head rest, as suggested by Donaldson and Cogan (1950). When the rabbit's cornea is being measured the animal is put under general anaesthesia and placed in a box which rotates about a pivot under its head so that both eyes may be conveniently observed in turn. This box is supported on a bracket which replaces the normal head rest.

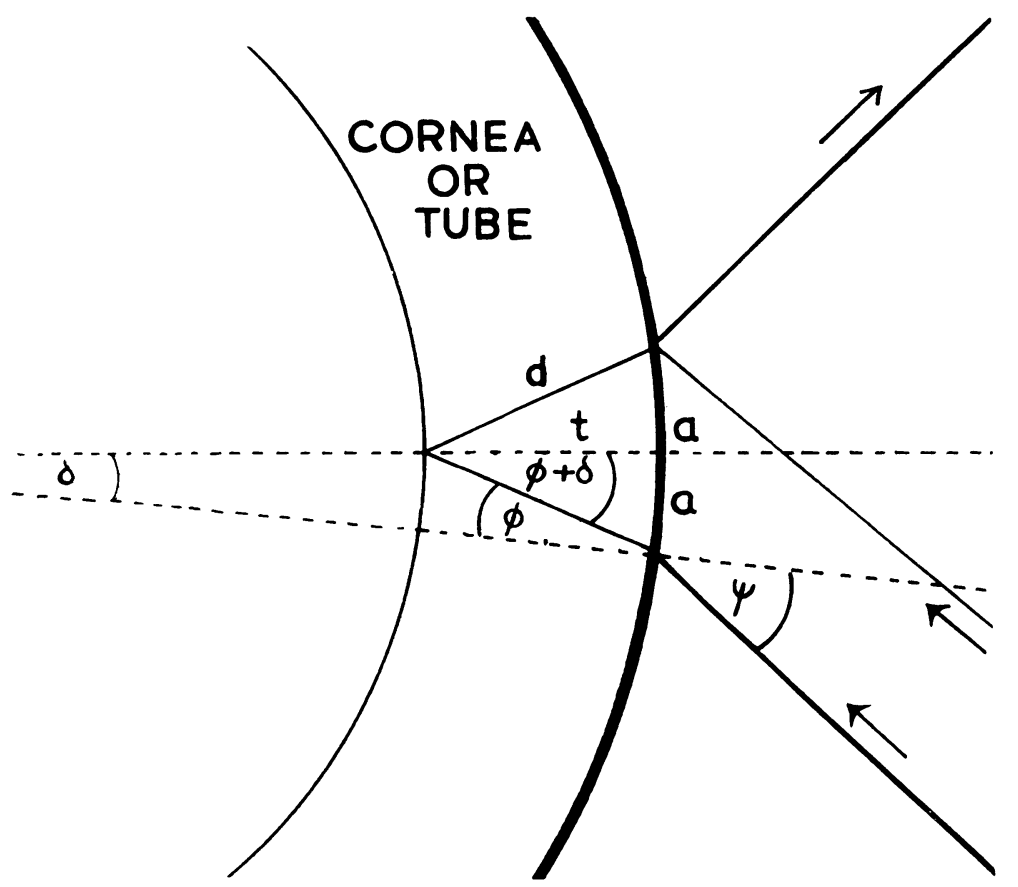

FIc. 3.- Principle of calibration. Path of rays in general case (cf. Fig. 2).

Calibration

The instrument was calibrated directly by measurements made upon glass and celluloid cylinders having a radius of curvature equal to that of the cornea. Where glass tubes are employed, they may be filled with paraffin oil and the determinations carried out without adjustment to the instrument. For the thinnest part of the calibration. air-filled celluloid tubes were necessary; the perspex plate was displaced to make the front and rear reflections equal in intensity, and a correction was made 
for the thickness of the coloured celluloid normally interposed in the beam. With these tubes it was possible to adjust the curvature and confirm that normal variations between different eyes would not significantly affect the calibration, as has been shown mathematically by von Bahr for his instrument. If the curvature in the horizontal plane of a lamina has a definite value, the setting of the instrument is determined by its optical thickness and is independent of its refractive index within normal limits. For in Fig. 3 (which represents a horizontal section and the correctly aligned reflected rays from the front and rear surfaces) we have:

$$
\begin{aligned}
n \sin \phi & =\sin \psi \\
t & =d \cos (\phi+\delta) \\
a & =d \sin (\phi+\delta)
\end{aligned}
$$

If $T$ is the optical thickness:

$$
\begin{aligned}
T & =\frac{t}{n} \\
& =\frac{a}{\sin \phi} \cdot \frac{\sin \phi \cos (\phi+\delta)}{\sin (\phi+\delta)} \text { from equations (1), (2), and (3). }
\end{aligned}
$$

Thus, if we have a glass tube with its radius of curvature that of the cornea so that $\psi_{g}=\psi_{c}$, and of such a thickness that they give the same readings on the apparatus, so that $a_{z}=a_{c}$,

$$
\begin{aligned}
\text { then: } \quad \frac{T_{c}}{T_{g}} & =\frac{\sin \left(\phi_{g}+\delta\right)}{\sin \phi_{g}} \cdot \frac{\sin \phi_{c}}{\sin \left(\phi_{c}+\delta\right)} \cdot \frac{\cos \left(\phi_{c}+\delta\right)}{\cos \left(\phi_{g}+\delta\right)} \\
\text { and, with } n_{c} & =1.376, \quad n_{g}=1.50, \quad t_{c}=0.5 \mathrm{~min} ., \quad \text { and } \psi=25^{\circ}, \\
\text { we obtain } T_{c} & =0.996 T_{g} .
\end{aligned}
$$

The optical thickness of the calibrating cylinders was measured directly by means of the fine focusing adjustment of a microscope, which was in turn calibrated by an engineer's dial-gauge accurate to $1 / 10,000$ in. The calibration was carefully checked by making determinations on two spherical, polished, perspex shells having curvatures and optical thicknesses within the range of the human cornea. The thickness was measured with the instrument at one point on each, and the shell was then rotated $180^{\circ}$ round the radius of the sphere passing through this point and another measurement made. By averaging the readings thus obtained by measuring in two opposite directions along the same meridian, allowance is made for any slight deviation from parallelism between the two faces. Confirmatory measurements round the meridian at right angles gave averages differing from the first by amounts corresponding to $0.000 \mathrm{~mm}$. and $0.008 \mathrm{~mm}$. in the two cases. The optical thickness was determined with the microscope, allowance being made for the curvature of the sphere, and was also calculated from the refractive index and the thickness as measured directly with the dial-gauge, the results agreeing within $0.005 \mathrm{~mm}$. It is thought that the overall error in calibration is unlikely to be as much as $0.01 \mathrm{~mm}$. though it may well be as much as $0.005 \mathrm{~mm}$. The true thickness was calculated from the optical thickness of the cornea, accepting the value $\mathbf{1 . 3 7 6}$ for its refractive index.

\section{Performance}

Although the instrument was designed for use with rabbits, its performance would be more readily compared with those previously 
described if it was given in respect of the human eye ; accordingly the results of detailed measurements in a few such eyes and representative measurements on a fair sized sample will be given below.

The scale of the instrument is easily read to $0.25^{\circ}$, which corresponds to about $0.005 \mathrm{~mm}$. in the range covered by the human cornea, that is about 1 per cent. of its total thickness. The self consistency of the readings was estimated by taking 25 determinations in one position on one eye, resulting in a standard deviation of $0.011 \mathrm{~mm}$. In general, for other eyes, four readings were taken in any one position, though more were added if any significant discordances appeared in the first group.

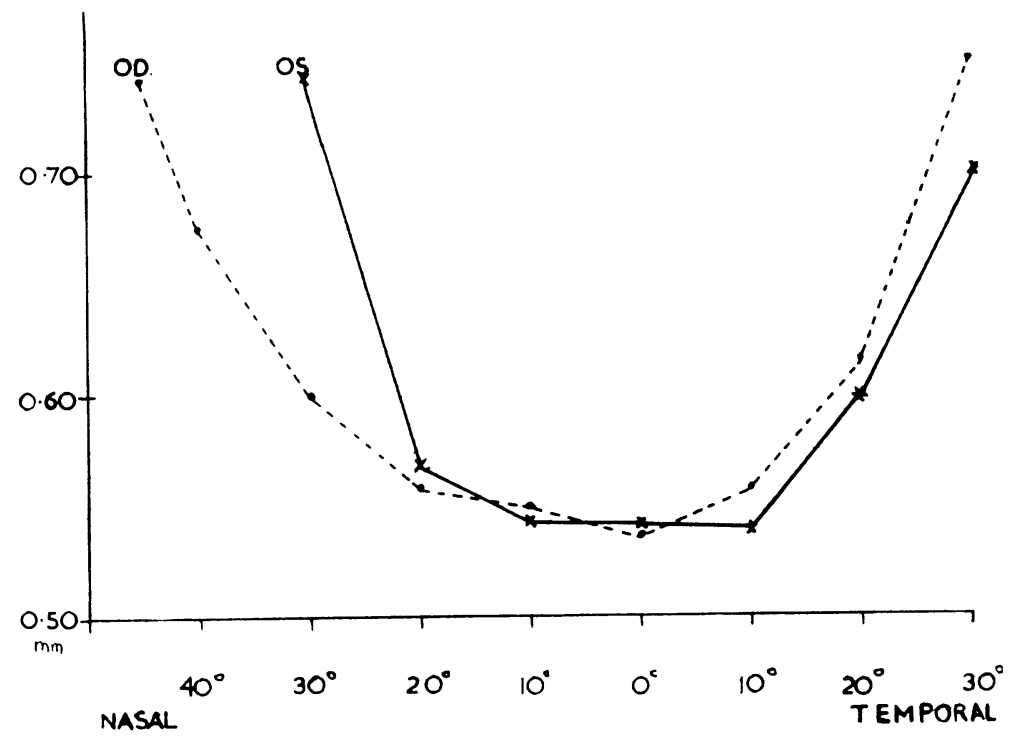

FIG. 4.-Variations in thickness of human cornea at various angles round equator (position of $0^{-}$estimated by observer).

In a few subjects, the corneal thickness was estimated at 10 intervals as far round the periphery as practicable. One typical instance is shown in Fig. 4. The readings in the extreme periphery are not accurate as the corneal surfaces are not parallel here. Nevertheless, the expected thickening of the cornea towards its edges is clearly shown, and, what is more important, a stretch of some $20^{\circ}$ or $3 \mathrm{~mm}$. in the centre where the thickness is minimal and appreciably constant. There should be no difficulty in estimating visually the middle point of the cornea to this degree of accuracy, and the $0^{\circ}$ point on this curve was derived by this simple procedure.

In the measurements on the main body of subjects which are described below, the initial measurement on an eye was always made in this way, and then two further estimations some 1 to $2 \mathrm{~mm}$. on 
either side of the first. Only in nine cases out of 88 was one of these estimates smaller than the first, and only in one case was it more than $0.01 \mathrm{~mm}$. smaller. The smaller estimate was accepted in each case. A determinative value for the thickness of a human cornea can therefore be obtained without the necessity of further refinement in the technique.

AVERAGe THICKNESS OF THE HUMAN CORNEA.-To find a representative value for the human eye, the corneae of 44 volunteers from the staff and students of this Institute, 24 male, and twenty female, were measured. All the females were aged between 18 and 30 years and all but five of the males between 20 and 35 years. Every

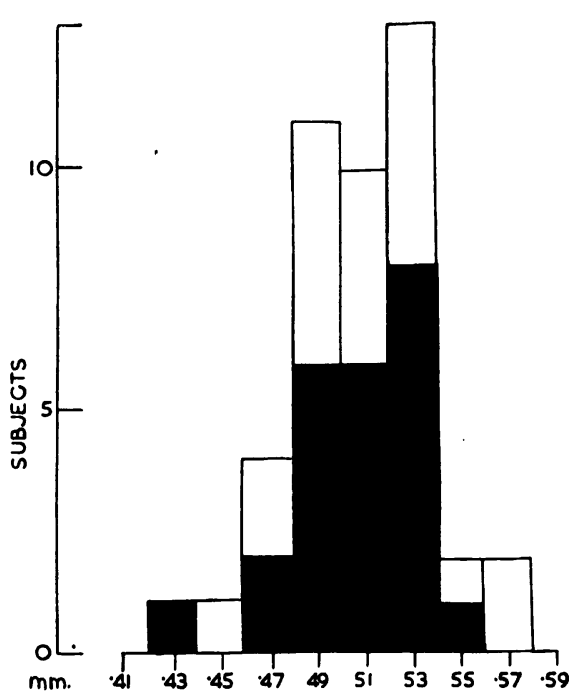

Fig. 5.-Distribution of corneal thickness. Black areas, men. White areas, women. subject had two healthy eyes and the majority were emmetropes or had corrections of two dioptres or less. Two of the men were 4-5 dioptre myopes and one woman needed a similar correction in one eye only.

The mean of the average thickness of each pair of eyes for this group is $0.507 \mathrm{~mm}$. \pm 0.0042 (standard error of mean of 44 subjects), $\sigma=0.028 \mathrm{~mm}$. The mean for the women is $0.004 \mathrm{~mm}$. higher than that for the men, this being insignificant in view of the estimated standard error of the difference of the means, $0.008 \mathrm{~mm}$. The dispersion for the women $(\sigma=$ 0.031 ), is rather higher than for men $(\sigma=0.026)$, but this also is not significant, since the estimated standard error for the difference in $\sigma$ is $0.006 \mathrm{~mm}$. The distribution of these results is shown as a histogram in Fig. 5. The average difference between left and right eyes is $0.0011 \pm 0.0011 \mathrm{~mm}$. and is not significant. The standard deviation of the difference between the two eyes $(0.0075 \mathrm{~mm}$.) is that which would be expected on the basis of four determinations on each cornea if pairs of corneae were equally thick, and this would appear to be the case.

Comparison with Previous Results.- The values given by previous observers are quoted extensively by von Bahr (1948a) and will not be transcribed. The largest set of observations are those of this author himself who obtained a mean value of $0.565 \mathrm{~mm}$. on 224 eyes of 125 
persons. The standard error of the mean is $0.0042 \mathrm{~mm}$., if, contrary to von Bahr, the number of people rather than the number of eyes is used as the size of the sample, as pairs of eyes correspond closely. The difference between his mean and ours is statistically very significant, though perhaps not important for practical purposes, either instrument being capable of detecting differences between its own value for the normal mean, and significantly different values for abnormal corneae. A small part of the difference could be due to his measurement being made in the centre of the pupil rather than at the point of minimum thickness of the cornea. If the entire difference were due to this, however, his dispersion should be much greater than ours, but as his standard deviation is only $0.047 \mathrm{~mm}$. there must be a systematic error in one method or the other.

Such errors should be less likely to occur if a direct method of calibration, as described here, is employed, and confirmation of our lower value is to some extent provided by the other workers using a variety of methods quoted by von Bahr. Only Blix (1880), Fincham, and Gullstrand (1924) give results whose dispersions are compatible with those of von Bahr and ourselves. The range of the values of Blix $(0.482$ to $0.576 \mathrm{~mm}$.), is all that is available to us, and this is not sufficient to effect a comparison. Fincham used the method of measuring the movement required to focus the slitlamp alternately on the back and front faces of the cornea. His figures (quoted by Koby, 1930) evidently make no allowance for the influence of the curvature of the cornea on its optical thickness. If this factor is included, the mean he obtains for nine adults is $0.508 \mathrm{~mm}$. \pm 0.012 , in excellent agreement with our figure, and if three children under 12 years of age are included the mean is $0.519 \mathrm{~mm}$. \pm 0.011 . Even if this second figure is adopted, the level of significance of the agreement of Fincham's results with ours and von Bahr's is 0.3 and 0.0003 respectively, assuming all distributions to be normal. Gullstrand made only two determinations ( 0.46 and $0.51 \mathrm{~mm}$.), which were obtained, however, by a very accurate method. The probability of these values belonging to our distribution rather than to von Bahr's is nine times greater.

The cause of a systematic error in von Bahr's instrument is not self-evident, though a latent inaccuracy is revealed by the misleading results it gives in measurements taken round the corneal equator. In the one example quoted, both corneae in one subject increase in thickness continuously from right to left instead of showing a central minimum.

\section{Summary}

A simple attachment to the Haag-Streit slit lamp which can be used to measure the thickness of the cornea in vivo is described and illustrated. 
On 44 human subjects the mean value found for the minimum corneal thickness was $0.507 \mathrm{~mm} . \pm 0.0042(\sigma=0.028)$.

Our thanks are due to Mr. C. Purvis for constructing this apparatus.

\section{REFERENCES}

VON BAHR, G. (1948a). Acta Ophthal., 26, 247.

(1948b). Trans. Ophthal. Soc. U.K., 68, 515.

Blix, M. (1880). Uppsala Läk Fören. Förh., 15, 349. Cited by von Bahr (1948a).

Donaldson, D. D., and Cogan, D. G. (1950). Arch. Ophthal., Chicago, 43, 555.

Fincham, E. F. Quoted by Koby (1930).

Gullstrand, A. (1924). In Helmholtz's “Treatise on Physiological Optics", ed. J. Southall, vol. 1, p. 327. Optical Society of America.

Kову, F. Е. (1930). Rev. gén. Ophtal., 44, 222. 